\section{Nachhaltigkeitseffekte durch Herstellung und Anwendung nanotechnologischer Produkte}

\author{
von Michael Steinfeldt, Institut für ökologi- \\ sche Wirtschaftsforschung
}

Mit Nanotechnologie werden gerade auch in Richtung Nachhaltigkeit große Erwartungen verbunden. In der Folge wird ein prospektiver Technologiebewertungs- und Gestaltungsansatz skizziert, die hiermit erreichten Ergebnisse vorgestellt und kritisch diskutiert. Mit nanotechnologischen Anwendungen können große Ökoeffizienzpotenziale erschlossen werden, Risikopotenziale dürfen aber auch nicht vernachlässigt werden. Die frühe Phase der Technologieentwicklung von Nanotechnologie bietet große Gestaltungspotenziale in Richtung Nachhaltigkeit.

\section{Einleitung}

Die Nanotechnologie wird vielfach als Basisinnovation bezeichnet, d. h. es wird erwartet, dass von ihr zahlreiche innovative Entwicklungen in den verschiedensten technologischen Bereichen und in den verschiedensten gesellschaftlichen Anwendungsfeldern und ökonomischen Branchen ausgehen.

Die weitreichenden und als potenziell machbar angesehenen Möglichkeiten der Nanotechnologien finden ihren Widerhall in z. T. extremen Bewertungen: auf der einen Seite eine radical green vision (Überwindung jeglicher Umweltbelastungen) und auf der anderen Seite die Gefahren der Unbeherrschbarkeit „der“ Nanotechnologie, insbesondere mit Blick auf langfristige Entwicklungspotenziale (vgl. Joy 2000; etc-group 2002).

Mit diesen typisierten Diskussionssträngen sind letztlich auch gesellschaftliche Kontroversen über die Entwicklungsrichtungen verbunden. Die Kontroverse betrifft dabei sowohl die ökologischen und ökonomischen, aber auch die sozialen Konsequenzen nanotechnologischer Entwicklungen und Visionen.
2 Das Projekt: „Nachhaltigkeitseffekte durch Herstellung und Anwendung nanotechnologischer Produkte"

Im Rahmen der vom BMBF geförderten „Innovations- und Technikanalyse zur Nanotechnologie“ bearbeitete das Institut für Ökologische Wirtschaftsforschung (IÖW) in Kooperation mit der Universität Bremen sowie zwei Praxispartnern die Themenstellung „Nachhaltigkeitseffekte durch Herstellung und Anwendung nanotechnologischer Produkte“ (FKZ 16I1504). Das Projekt griff den aktuellen Stand der Stoff- und Technikbewertung auf und versuchte, ihn in Richtung einer integrierten Nachhaltigkeitsbewertung weiter zu entwickeln. Dabei lag der Fokus auf den ökologischen Chancen und Risiken dieser sich entwickelnden Technologie. Dem Projekt stellten sich zwei zentrale Fragen:

1. Wie kann es gelingen, die erwartbaren Wirkungen einer erst im Entstehen begriffenen Technologie abzuschätzen, und

2. Wie kann erfolgreich auf die nachhaltigkeitsorientierte Gestaltung der Nanotechnologie Einfluss genommen werden?

Wenn die Anwendungsfelder einer Technologie noch weitgehend offen sind und zudem mit noch unbekannten neuen Wirkungen gerechnet werden muss, bleibt als wesentliche zu untersuchende Variable die Technologie selbst. Es empfiehlt sich also eine Blickwende von den „Wirkungen“" zum „Bewirkenden“, zur Analyse und Charakterisierung der Nanotechnologie. Zum anderen wird damit zugleich die Basis gelegt für die Analyse potenzieller oder in der Entwicklung befindlicher Anwendungskontexte. Schließlich entstehen Technologien nicht naturwüchsig, vielmehr werden sie bewusst oder unbewusst im Kontext von Innovationssystemen gestaltet. Diese Gestaltungspotenziale gilt es bspw. im Kontext der Entwicklung von Leitbildern - wie z. B. „nachhaltige Nanotechnologien" - zu nutzen.

Um der Komplexität der Aufgabenstellung gerecht zu werden, wurde im Projekt ein dreistufiger Ansatz zur prospektiven Technologiebewertung und -gestaltung von Nanotechnologie verfolgt. 
1. Ansatz-prospektiv

Bewertung der Nanotechnologie und ihrer Wirkungen durch eine Charakterisierung der Technologie

2. Ansatz-prozessbegleitend

Bewertung von Nachhaltigkeitseffekten an konkreten Anwendungsbeispielen im Vergleich $\mathrm{zu}$ bestehenden Produkten und Verfahren

3. Ansatz-gestaltend

Leitbilder als Steuerungs,--instrumente" in der Technikgestaltung, Einbeziehung der kurzfristigen/langfristigen Perspektive.

\section{Charakterisierung der Nanotechnologie}

Jede Form der Technikfolgenabschätzung hat mit dem Prognoseproblem, mit dem Umgang mit Nichtwissen (Noch-Nicht-Wissen bzw. Nicht-Wissbarkeit) und Unsicherheit zu kämpfen. Der prospektiv orientierte Ansatz fokussiert auf die Bewertung der Nanotechnologie und ihrer Wirkungen durch eine „Charakterisierung der Technologie" (ausführlich hierzu Gleich 2004). Die Problematik des Nichtswissens bei neuen Technologieentwicklungen bewusst und konsequent reflektierend, ermöglicht die Technologiecharakterisierung, mögliche Gefährdungspotenziale und positive Effekte ,abzuleiten“ und aufzuzeigen.

Die Nanotechnologie ist in erster Linie durch die Dimension charakterisiert, in der sie sich bewegt: In der Nanowelt bewegen wir uns auf der Ebene einzelner Moleküle und Atome in der Größe eines Millionstel Millimeters. Das Besondere an dieser Dimension ist nun, dass Nanopartikel sich meist völlig anders verhalten als ihre grobkörnigeren Pendants. So führt bspw. die große spezifische Oberfläche von Nanopartikeln in der Regel zu einer Steigerung der chemischen Reaktivität bzw. der katalytischen Aktivität. Die relativ geringe Anzahl von Atomen in Nanopartikeln hebt andererseits den quasi kontinuierlichen Energiezustand der Elektronen im Festkörper auf und führt zu abweichenden optischen, elektrischen und magnetischen Eigenschaften.

Ausgehend von diesen Grundcharakteristika der Nanotechnologie können in einem nächsten Schritt mögliche positive Effekte und Potenziale bzw. mögliche und erwartbare problematische Wirkungen „abgeleitet“ werden (vgl. Tab. 1).

Aus dieser Charakterisierungsübersicht wird deutlich, dass sich derzeit viele Gefährdungspotenziale auf Grund der neuen Qualitäten von Nanopartikeln ergeben können, die besonders beim offenen Umgang mit ihnen zum Tragen kommen würden. Dieses Thema, welches auch im aktuellen Diskurs zu Risiken die höchste Priorität besitzt, wurde daher in einer vertiefenden Fallstudie gesondert bearbeitet.

Bei der Bewertung von Risikogesichtspunkten weiter gehender, teilweise weit in der Zukunft liegender Anwendungsfelder von Selbstorganisation (z. B. Kombination von Nanotechnologie und Biotechnologie, Nanotechnologie und Robotik) erlangt der Aspekt einer möglichen Selbstreproduktion eine viel stärkere Bedeutung. Mit der Fähigkeit zur Selbstreproduktion, wie sie z. B. gentechnisch veränderte Organismen besitzen, dürften auf jeden Fall neuartige Risikodimensionen bezüglich Gesundheits- und Umweltgefährdungen eröffnet werden, während das für reine Selbstorganisationsprinzipien weniger gilt. Solange sich die Nanotechnologie auf den Umgang mit Molekülen beschränkt, dürfte ein solcher Schritt von der Selbstorganisation von Molekülen zur Selbstreproduktion und Vervielfältigung von Robotern oder Organismen - wenn er denn nicht bewusst angestrebt wird - eher unwahrscheinlich sein. Auf der Grundlage einer Verschmelzung der Nanotechnologie mit der gentechnologischen Manipulation von zur Selbstreproduktion fähigen Organismen könnte ein solcher Schritt allerdings realisierbar werden.

Neben dieser Charakterisierung der Nanotechnologie auf Technologieebene wurden in einem nächsten Schritt die bisher relevanten nanotechnologischen Herstellungsverfahren (Gasphasenabscheidung, Flammenverfahren, Sol-Gel-Verfahren, Fällung, Molekulares Prägen, Lithographie, Selbstorganisation) hinsichtlich ihres technologischen und damit in Verbindung stehenden energetischen Aufwandes sowie hinsichtlich ihrer Risikopotenziale für die Freisetzung von Nanopartikeln qualitativ bewertet. 
Tab. 1: Nanoqualitäten und dadurch erwartbare positive ökologische Effekte und Potenziale bzw. problematische Wirkungen

\begin{tabular}{|c|c|c|c|}
\hline Nanoqualität & & $\begin{array}{l}\text { + Positive ökologische Effekte und Po- } \\
\text { tenziale/ } \\
\text { - Probleme und Gefährdungspotenziale }\end{array}$ & Bewertungsansätze \\
\hline \multirow[t]{2}{*}{$\begin{array}{l}\text { Kleinheit und Mobilität der } \\
\text { Partikel }\end{array}$} & + & $\begin{array}{l}\text { Gezielte Nutzung für ressourcen-/ökoeffi- } \\
\text { ziente Technik }\end{array}$ & \multirow{2}{*}{$\begin{array}{l}\text { Ökobilanz, } \\
\text { Ausbreitungs- und Expositionsmodelle, } \\
\text { (öko-)toxikologische Prüfung, } \\
\text { Tierversuche, Epidemiologie }\end{array}$} \\
\hline & - & $\begin{array}{l}\text { Lungen- bzw. alveolengängig } \\
\text { Durchgang durch Zellmembranen, Blut- } \\
\text { Hirn-Schranke } \\
\text { Mobilität, Persistenz und Löslichkeit als } \\
\text { Indizien für Bioakkumulation und Umwelt- } \\
\text { gefährdung }\end{array}$ & \\
\hline \multirow[t]{2}{*}{$\begin{array}{l}\text { Definiertheit, Korn-/ } \\
\text { Schichtgrößen, Reinheit }\end{array}$} & + & $\begin{array}{l}\text { Gezielte Nutzung für ressourcen- / ökoeffi- } \\
\text { ziente Technik }\end{array}$ & \multirow[t]{2}{*}{$\begin{array}{l}\text { Ökobilanz, Entropiebilanz, } \\
\text { Frage nach ,ökologischer Amortisierung, }\end{array}$} \\
\hline & - & $\begin{array}{l}\text { Erhöhter Herstellungsaufwand, höhere } \\
\text { Stoff- und Energieströme, erhöhter Res- } \\
\text { sourcenverbrauch }\end{array}$ & \\
\hline \multirow[t]{2}{*}{ Stoffqualität } & + & $\begin{array}{l}\text { Möglicher Ersatz gesundheits- und umwelt- } \\
\text { gefährdender Stoffe }\end{array}$ & \multirow{2}{*}{$\begin{array}{l}\text { Toxikologie, Ökotoxikologie, } \\
\text { Verhältnis zwischen ,natürlichen’ und } \\
\text {,anthropogenen' Stoffumsätzen }\end{array}$} \\
\hline & - & $\begin{array}{l}\text { Gesundheits- und Umweltgefährdung durch } \\
\text { problematische (seltene) Elemente oder } \\
\text { Stoffgruppen im umweltoffenen Einsatz }\end{array}$ & \\
\hline \multirow[t]{2}{*}{$\begin{array}{l}\text { Adhäsion, Kohäsion, } \\
\text { Agglomeration }\end{array}$} & + & $\begin{array}{l}\text {,Eigensicherheit' durch Tendenz zur Adhä- } \\
\text { sions-, Kohäsions- und Agglomeration von } \\
\text { Nanopartikeln }\end{array}$ & \multirow{2}{*}{$\begin{array}{l}\text { Ausbreitungs- und Expositionsmodelle, } \\
\text { (öko-)toxikologische Prüfung, } \\
\text { Tierversuche, } \\
\text { Epidemiologie, } \\
\text { Atmosphärenchemie, } \\
\text { Risikoanalyse }\end{array}$} \\
\hline & - & $\begin{array}{l}\text { Verhalten ,freigesetzter' Nanopartikel oder } \\
\text { Nanofasern in der Umwelt, } \\
\text { Mobilisierende und einschleusende Wir- } \\
\text { kung von Nanopartikeln auf Toxine oder } \\
\text { Schwermetalle (Huckepack) }\end{array}$ & \\
\hline \multirow[t]{2}{*}{$\begin{array}{l}\text { Neue chemische Effekte, } \\
\text { verändertes Verhalten }\end{array}$} & + & $\begin{array}{l}\text { Nutzung des veränderten Verhaltens für } \\
\text { ressourcen-/ökoeffiziente Technik, z. B. } \\
\text { Nutzung der katalytischen Wirkungen für } \\
\text { effizientere chemische Prozesse oder im } \\
\text { Umweltbereich }\end{array}$ & \multirow[t]{2}{*}{$\begin{array}{l}\text { Ökobilanz, } \\
\text { Ausbreitungs- und Expositionsmodelle, } \\
\text { (öko-)toxikologische Prüfung, } \\
\text { z. B. auch auf Allergie/Sensibilisierung, } \\
\text { Tierversuche, Epidemiologie, } \\
\text { Atmosphärenchemie, } \\
\text { Risikoanalyse }\end{array}$} \\
\hline & - & $\begin{array}{l}\text { Veränderungen bei: Löslichkeit, Reaktivi- } \\
\text { tät, Selektivität, katalytische Wirkung, } \\
\text { photokatalytische Wirkung, Temperaturab- } \\
\text { hängigkeit von Phasenübergängen lassen } \\
\text { überraschende technische, chemische, } \\
\text { toxische und ökotoxische Effekte erwarten }\end{array}$ & \\
\hline \multirow[t]{2}{*}{$\begin{array}{l}\text { Neue physikalische Effekte, } \\
\text { verändertes optisches, } \\
\text { elektrisches, magnetisches } \\
\text { Verhalten }\end{array}$} & + & $\begin{array}{l}\text { Gezielte Nutzung der Effekte bzw. verän- } \\
\text { derten Eigenschaften für ressourcen-/öko- } \\
\text { effiziente Technik, z. B. } \\
\text { GMR-Effekt, Tyndell-Effekt, Quanten- } \\
\text { Effekte, Tunneleffekt }\end{array}$ & \multirow[t]{2}{*}{$\begin{array}{l}\text { Ökobilanz, } \\
\text { Für technische Systeme: } \\
\text { FMEA, } \\
\text { Fehlerbaumanalyse }\end{array}$} \\
\hline & - & $\begin{array}{l}\text { Meist auf hochreine und hochdefinierte } \\
\text {,technische Umgebungen' angewiesen. } \\
\text { Dort sind (bei Nichteinhaltung) Überra- } \\
\text { schungen erwartbar (technisches Versagen). }\end{array}$ & \\
\hline \multirow[t]{2}{*}{ Selbstorganisation } & + & $\begin{array}{l}\text { Gezielte Nutzung für ressourcen-/ökoeffi- } \\
\text { ziente und konsistente Technik }\end{array}$ & \multirow[t]{2}{*}{$\begin{array}{l}\text { Risikoanalyse, Eingriffstiefe, Ökobilanz, } \\
\text { Umweltverträglichkeitsprüfung, Szenario- } \\
\text { technik }\end{array}$} \\
\hline & - & $\begin{array}{l}\text { Gefahr unkontrollierter Entwicklungen, } \\
\text { selbstreplizierende Nanobots }\end{array}$ & \\
\hline
\end{tabular}

Ouelle: verändert nach (Gleich 2004) und (Steinfeldt 2003) 


\section{Bewertung konkreter Anwendungskon- texte - Ökobilanzierung}

Aufbauend auf dieser Charakterisierung der Nanotechnologie und ihrer bisherigen Herstellungsverfahren verfolgte der prozessbegleitende Bewertungsansatz die Ermittlung von Nachhaltigkeitseffekten an konkreten Anwendungsbeispielen im Vergleich zu bestehenden Produkten und Verfahren, wobei der Fokus auf die ökologischen Chancen und Risiken gerichtet war.

Als Bewertungsansatz orientierten sich die erstellten ökologischen Profilbetrachtungen an der Methodik der Ökobilanzierung. Die Ökobilanz ist die am weitesten entwickelte und normierte Methode zur Abschätzung der mit einem Produkt verbundenen Umweltaspekte und produktspezifischen potenziellen Umweltwirkungen. Ein Vorteil besteht darin, dass durch die Ökobilanz die Analyse von Ökoeffizienzpotenzialen im Vergleich zu bestehenden Anwendungen möglich ist. Auf der anderen Seite besitzt die Ökobilanzmethode Defizite; so existieren bisher nicht für alle Wirkungskategorien allgemein akzeptierte Wirkungsmodelle. Dies muss insbesondere für die relevanten Kategorien Humantoxizität und Ökotoxizität festgestellt werden. So geht die Berücksichtigung der Belastung durch Feinstäube (PM10-Risiko thematisiert ein mögliches Toxizitätspotenzial durch Partikel $<10 \mu \mathrm{m}$ ) in Ökobilanzen bei Nanotechnologieanwendungen allein schon größenordnungsmäßig am Ziel vorbei. Außerdem werden in Ökobilanzen Risiken sowie die Wirkmächtigkeit von Anwendungen nicht betrachtet. Ein umfassendes Methodenset müsste derartige Analysen mit beinhalten.

Im Projekt wurde mit dieser Ambivalenz dahingehend umgegangen, dass bei der Auswahl der konkreten Anwendungskontexte bewusst Schwerpunkte gesetzt wurden. Aus dem Spektrum an nanotechnologischen Anwendungen wurden einerseits vier Fallbeispiele mit potenziell erwartbaren Ökoeffizienzpotenzialen gezielt auf Grundlage einer Sichtung und qualitativen Bewertung ausgewählt. Zudem wurden mögliche Risiko- und Gefährdungspotenziale nanotechnologischer Anwendungen fokussiert am Thema Nanopartikel analysiert und diskutiert (vgl. Tab. 2).
Tab. 2: Übersicht der untersuchten Fallstudien

\begin{tabular}{|l|l|}
\hline Anwendungskontexte & Zielstellung \\
\hline Ökoeffiziente Nano- & $\begin{array}{l}\text { Darstellung des Ökoeffizienz- } \\
\text { potenzials von Nanobeschich- } \\
\text { tungen in Form eines verglei- } \\
\text { chenden Ökoprofils } \\
\text { (Nanolack auf Basis der Sol- } \\
\text { Gel-Technologie im Vergleich } \\
\text { zu Wasserlack, Lösemittellacken } \\
\text { und Pulverlack) }\end{array}$ \\
\hline $\begin{array}{l}\text { Nanotechnologische } \\
\text { Prozessinnovation der } \\
\text { Styrolsynthese }\end{array}$ & $\begin{array}{l}\text { Darstellung des Ökoeffizienz- } \\
\text { potenzials von Nanotechnologie } \\
\text { in einer katalytischen An- } \\
\text { wendung in Form eines ver- } \\
\text { gleichenden Ökoprofils (Nano- } \\
\text { tube-Katalysator im Vergleich } \\
\text { zu Katalysator auf Eisenoxidba- } \\
\text { sis) }\end{array}$ \\
\hline $\begin{array}{l}\text { Nanoinnovationen im } \\
\text { Displaybereich }\end{array}$ & $\begin{array}{l}\text { Abschätzung möglicher Ökoef- } \\
\text { fizienzpotenziale der Display- } \\
\text { innovationen durch qualitativen } \\
\text { Vergleich } \\
\text { (OLED - Organic Light Emitter } \\
\text { Display und Nanoröhren- } \\
\text { Feldemitterdisplays im Ver- } \\
\text { gleich zu Kathodenstrahlröhre, } \\
\text { Flüssigkristallbildschirm und } \\
\text { Plasmabildschirm) }\end{array}$ \\
\hline $\begin{array}{l}\text { Risikopotenziale } \\
\text { nanotechnologischer }\end{array}$ \\
Anwendungen & $\begin{array}{l}\text { Darstellung des Ökoeffizienz- } \\
\text { potenzials von Nanoanwen- } \\
\text { dungen im Lichtbereich in } \\
\text { Form eines vergleichenden } \\
\text { Ökoprofils } \\
\text { (Weiße LED und Quanten Dots } \\
\text { im Vergleich zu Glühlampe } \\
\text { und Kompaktleuchtstofflampe) }\end{array}$ \\
$\begin{array}{l}\text { Exemplarische Diskussion } \\
\text { möglicher Risiko- und Gefähr- } \\
\text { dungspotenziale unter anderem } \\
\text { am Beispiel Titandioxid, weni- } \\
\text { ger Betrachtung der Um- } \\
\text { weltauswirkungen }\end{array}$ \\
\hline
\end{tabular}

Im Ergebnis der ökobilanziellen Vergleiche ist festzuhalten, dass nanotechnologische Anwendungen nicht per se mit hohen ökologischen Entlastungspotenzialen verbunden sind. Gleichwohl konnten für die Mehrzahl der gezielt ausgewählten Anwendungskontexte hohe Ökoeffizienzpotenziale mit der gewählten Methode der vergleichenden Betrachtung von Funktionalitäten ermittelt werden.

Die Belastbarkeit der ermittelten Zahlen ist natürlich abhängig von der vorliegenden Qualität und Verfügbarkeit der Stoff- und Energiedaten für die einzelnen Anwendungen. Für noch in Entwicklung befindliche Nanoinnovationen sind 
nur schwer quantitative Aussagen für den Herstellungsaufwand zu treffen, wohingegen Aussagen für die Gebrauchsphase (meist zum Energieeinsparpotenzial) oft abschätzbar sind.

Im Bereich Beschichtungen von Aluminiumoberflächen konnte an Hand eines schon einsetzbaren Nanolackes eindrucksvoll gezeigt werden, dass durch den Einsatz von nanotechnologiebasierten Beschichtungen bei allen betrachteten Emissionen und Umweltwirkungen sehr hohe Ökoeffizienzpotenziale bestehen. Durch die geringe notwendige Beschichtungsdicke bei gleicher Funktionalität wird eine um den Faktor fünf höhere Ressourceneffizienz erreicht; Vorteile aus der Gebrauchsphase sind insbesondere im Transportsektor im Zuge des Leichtbautrends zu erwarten. Auch im Rahmen der Fallstudie Nanotechnologische Prozessinnovation der Styrolsynthese konnte aufgezeigt werden, dass durch den Einsatz eines nanostrukturierten Katalysators auf Basis von Nanotubes für den chemischen Prozess der Styrolsynthese auf der Prozessebene eine sehr hohe Energieeffizienzsteigerung erreichbar ist, die im Bereich von fast $50 \%$ liegen könnte. Bezogen auf den gesamten Produktlebensweg bis zum Styrol würde dies eine Effizienzsteigerung beim Energiebedarf von ca. $8-9 \%$ bedeuten.

In der Fallstudie Nanoinnovationen im Displaybereich war es schwerer, auf Basis der vorliegenden Daten konkrete Abschätzungen zu möglichen Ökoeffizienzpotenzialen zu formulieren, wobei auch hier sehr viel für Steigerungen der Material- und Energieeffizienz spricht. Z. B. würden sich OLEDs nach Überwindung der Probleme mit der Langzeitstabilität der organischen Leuchtstoffe gegenüber den vorherrschenden LCDs durch geringeren Herstellungsaufwand auszeichnen. Außerdem wird für die Gebrauchsphase eine um den Faktor 2 bessere Energieeffizienz erwartet. Über den gesamten Lebensweg erscheint damit eine 20 \%ige Energieeinsparung gegenüber LCD möglich.

Zentraler Maßstab für die ökologische Beurteilung von Lichtquellen für Beleuchtungszwecke sind der Energieverbrauch in der Gebrauchsphase und die damit verbundenen Emissionen. Hierbei zeigte sich, dass die heutige Weiße LED zwar gegenüber der klassischen Glühlampe besser abschneidet, aber im Vergleich zur Energiesparlampe um den Faktor 3 im Nachteil ist. Erst weiterentwickelte nano- technologiebasierte Produkte mit bedeutend höheren Lichtausbeuten werden mit anderen energiesparenden Lichtquellen in Konkurrenz treten können.

Für viele Bereiche sind aber recht eindeutige positive Aussagen hinsichtlich erwartbarer Ökoeffizienzpotenziale möglich. Die begleitende und prospektive Ökobilanzierung stellt ein durchaus brauchbares Instrument dar, um wesentliche Aspekte „nachhaltiger“" Technologieentwicklung zu identifizieren.

In der Fallstudie Risikopotenziale nanotechnologischer Anwendungen lag der Fokus auf den Wirkungen von Nanopartikeln und damit auf der Analyse und Diskussion potenzieller Risiken insbesondere durch Nanopartikel. Das Verhalten von Nanopartikeln unterscheidet sich von Materie auf der Makro-Ebene (vgl. Kap. 3: Charakterisierung der Technologie). Die analysierten Studien verweisen einerseits auf z. T. überraschendes Verhalten, andererseits geben sie Hinweise auf toxische Wirkungen von Nanopartikeln auf die Umwelt und menschliche Gesundheit. Besondere Bedeutung in Bezug auf toxische Wirkungen kommen in den Studien den Nanotubes und Buckyballs zu.

Alle bisher produzierten wissenschaftlichen Erkenntnisse sind aber vorläufig, teilweise widersprüchlich und beziehen sich nur auf einen Bruchteil von möglichen Effekten. Gleichzeitig scheint die Übertragbarkeit des bisher gewonnenen Wissens gering, verallgemeinerbare Aussagen und Klassifikationen zur Toxizität von Nanopartikeln können derzeit nicht getroffen werden.

Im Hinblick auf Toxizität und dem Verhalten von Nanopartikeln in der Umwelt besteht somit ein erheblicher Forschungsbedarf. Unter Berücksichtigung der Produktionsverfahren und der meisten Anwendungen erscheint aus heutiger Sicht das Problem der Nanopartikel begrenzt zu sein, da einerseits die Produktionsverfahren zumeist in wässrigen Lösungen oder in geschlossenen Systemen stattfinden. In einer Vielzahl von Produkten sind Nanopartikel fest eingebunden und stellen damit vermutlich kein Problem dar. Über den gesamten Lebenszyklus bestehen gleichwohl große Wissenslücken. 


\section{Gestaltende Ansätze für eine nachhaltige Nanotechnologie}

Technologieentwicklung ist nicht oder doch nur sehr begrenzt durch politische Interventionen steuerbar, vielmehr ergibt sich aus dem Zusammenwirken unterschiedlichster Akteure eine Pfadentwicklung der Technologie, die gestaltend begleitet werden kann. Die Bedeutung der frühzeitigen Identifikation adverser Effekte auf Umwelt- und Gesundheit wird durch die folgende Graphik dargestellt (vgl. Abb. 1).
Möglichkeit, potenzielle Umwelt- und Gesundheitsrisiken zu vermeiden.

Die Phase der Gestaltung von Produktionsprozessen und Produkten ist bereits in dem Sinne präformiert, als sie die FuE zur Grundlage ihrer Entwicklung haben. Die Prozess- und Produktgestaltung hat gleichwohl noch relativ viele Freiheitsgrade, die entscheidend für den Aspekt der „Eigensicherheit“ und die Belastungen in den Folgephasen sind. Die Gestaltungsoptionen sind begrenzter, gleichwohl in großem Umfang noch vorhanden.

Abb. 1: Zeitfenster der Gestaltung im Lebenszyklus

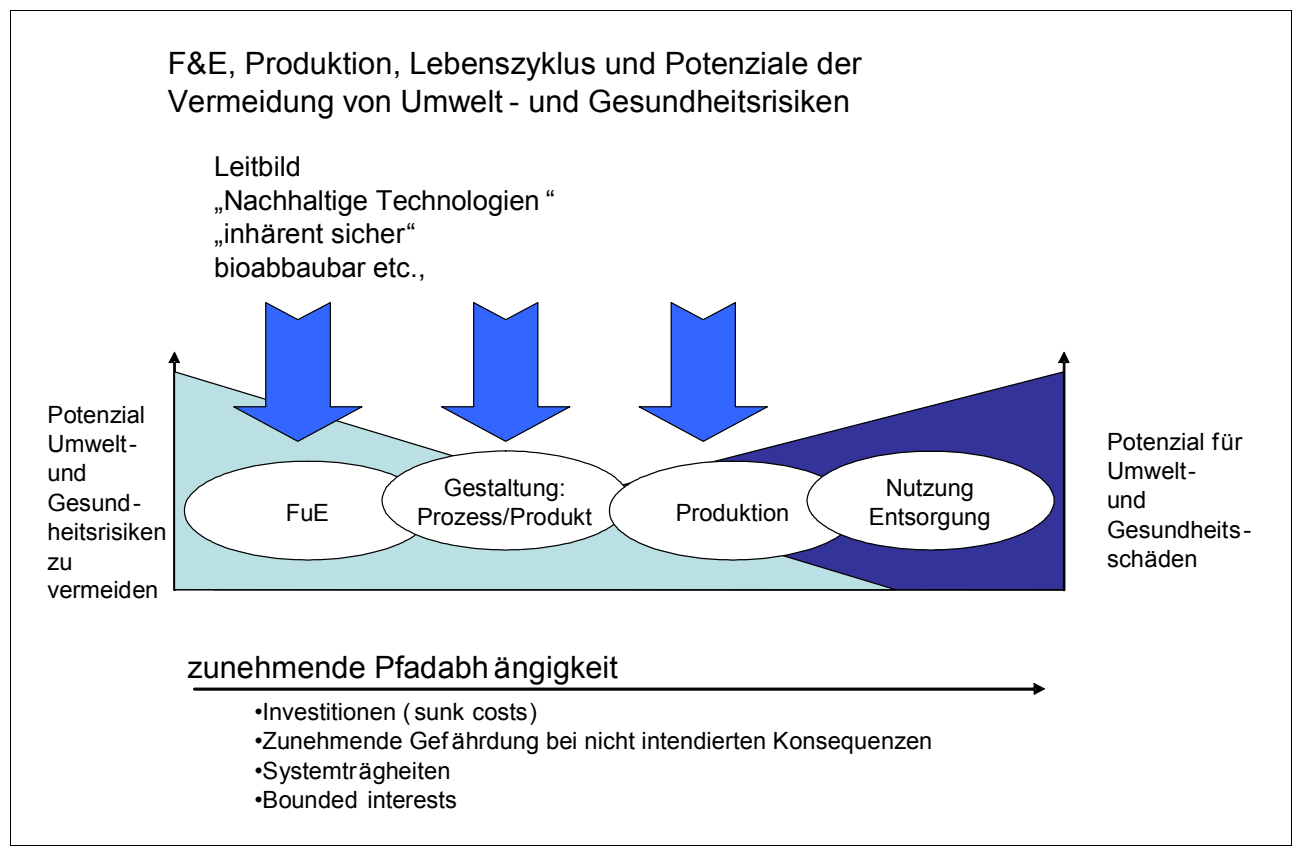

Quelle: Haum et al. 2004

Die Graphik verdeutlicht, dass entlang des gesamten Prozesses von der Grundlagenforschung über die angewandte Forschung, der Gestaltungs-, Nutzungs- und Entsorgungsphase phasentypische Vorsorgeoptionen entwickelt und generiert werden können und müssen, die jeweils auch als Folgenforschung charakterisiert werden können. In den unterschiedlichen Phasen sind jeweils unterschiedliche Akteure (mit-)verantwortlich. Dies kann bereits in der Phase der Grundlagenforschung beginnen (Wissenschaftsfolgenforschung), deren Ergebnisse in der Folge zu Forschungs- und Entwicklungsbemühungen im Bereich der angewandten Forschung führen. Nach unserer Auffassung besteht in diesen Phasen die größte
Die Gestaltungsoptionen im Produktionsprozess und während der Nutzung bis schließlich der Entsorgung nehmen deutlich ab, in der Regel können zu diesem Zeitpunkt alleine additive Maßnahmen noch greifen, indem bspw. die Prozesse und Produkte im Sinne der Sicherheitsdatenblätter gehandhabt werden.

Neben der wissenschaftlichen und technischen Pfadgestaltung ist zudem der Aspekt von Bedeutung, der von den getätigten Investitionen und von dem gebundenen Know-how und Wissen ausgeht - auch hier sind Pfadverfestigungen im Zeitablauf festzustellen. Bspw. hängt die Ausgestaltung dieser Pfade auch von Leitbildern ab. Leitbilder können eine Steuerungswirkung entfalten und zur Richtungssicherheit der Innovationen beitragen. Drei konkrete Leitbilder 
einer nachhaltigen Nanotechnologie mit unterschiedlichen Reichweiten wurden im Projekt entwickelt: „Ressourceneffiziente Nanotechnologie“, „Konsistente und eigensichere Nanotechnologie" sowie das langfristig orientierte Leitbild "Nanobionik“ (ausführlich hierzu Gleich 2004). Weitere Gestaltungsansätze und -instrumente wurden skizziert:

- Nachhaltiges Nanodesign in Forschung und Entwicklung

- Integration von Sicherheits-, Gesundheitsund Umweltschutzaspekten in das Wertschöpfungsketten übergreifende Qualitätsmanagement

- Staatliche Regulierungsansätze.

Darüber hinaus wurde auf die Wichtigkeit eines Prozesses der weiteren Technologiebegleitung und -gestaltung der Nanotechnologie verwiesen und einzelne Verfahrensvorschläge in Anlehnung an Konzepten wie Constructive Technology Assessment (CTA) (Rip et al. 1995) und real-time TA (Guston und Sarewitz 2001) erarbeitet.

\section{Fazit und Handlungsbedarfe}

Die dreistufige Vorgehensweise im Rahmen des Projektes konnte durch den Ansatz der Charakterisierung der Technologie wesentliche potenzielle Problembereiche von Nanotechnologien identifizieren, durch die Ökobilanzierung konnten mögliche Effizienzpotenziale der Anwendung der Nanotechnologien aufgezeigt werden, schließlich wurden Ansätze der weiteren Gestaltung aufgezeigt. Wesentliche Schlussfolgerungen, die aus der Studie gezogen werden konnten, sind, dass schon heute von Nanopartikeln in offenen Anwendungen ein nicht zu vernachlässigendes Risikopotenzial ausgeht. Die Ergebnisse der Ökobilanzierung zeigen, dass deutliche Umweltentlastungspotenziale erschlossen werden können, dies gilt aber nicht durchgehend in allen Anwendungsbereichen. Die begleitende Ökobilanzierung kann sich damit als ein wichtiges Instrument der nachhaltigkeitsorientierten Gestaltung bewähren. Schließlich ist darauf zu verweisen, dass den leitbildorientierten Gestaltungsansätzen, nicht zuletzt aufgrund der Erkenntnisse der ersten beiden Ansätze, eine wichtige Rolle für die weitere Technologieentwicklung in Richtung Nachhaltigkeit zukommt.
Bezogen auf die Bewertung von Risiken von Nanotechnologie besteht Forschungsbedarf bzgl.

- toxikologischer und ökotoxikologischer Untersuchungen,

- des Verhaltens der Nanopartikel in der Umwelt und deren Systematisierung,

- integrierter Untersuchungsprogramme.

Über die im Projekt untersuchten Fälle hinaus, die sich sehr stark auf anorganische Anwendungskontexte fokussierten, besteht insbesondere weiterer Forschungsbedarf

- im Bereich der Anwendungsfelder von Selbstorganisation im Hinblick auf die zu erwartenden Ökoeffizienzpotenziale,

- im Bereich der weiter gehenden Anwendungsfelder von Selbstorganisation (z. B. Kombination von Nanotechnologie und Biotechnologie, Nanotechnologie und Robotik) im Hinblick auf potenzielle Risiken (Frage des schleichenden Übergangs von Selbstorganisation zur Selbstreproduktion).

Darüber hinaus würden Ökoprofilbetrachtungen von nanotechnologischen Anwendungen wesentlich erleichtert, wenn Ökobilanzdaten für die relevanten Herstellungsverfahren öffentlich bereitstehen würden. Untersuchungen dazu liegen nicht vor und waren im Rahmen dieser Studie auch nicht möglich.

Die weitere Begleitung der Prozesse der Technologieentwicklung erscheint erforderlich, zum einen aus Gründen der Analyse potenzieller Risiken und der Auslotung von Chancen und zum anderen nicht zuletzt aufgrund der der Nanotechnologie zugeschriebenen ökonomischen und gesellschaftlichen Veränderungspotenziale.

Auf der instrumentellen Ebene erscheinen die bekannten Kommunikationsinstrumente im Grundsatz geeignet zu sein, um Handlungsorientierungen gerade auch für kleine und mittlere Unternehmen zu generieren (Leitfäden, Gestaltungsorientierungen etc.).

- Die frühe Phase der Technologieentwicklung bietet im Grundsatz große Gestaltungspotenziale in Richtung Nachhaltigkeit - diese müssen erschlossen werden. Leitbildentwicklung in gestaltender Absicht bietet sich an. 
Nanobionikprojekte zur Ausgestaltung des gleichnamigen Leitbildes sind zu fördern.

- Begleitende und gestaltungsorientierte Prozesse müssen vorangebracht werden (bspw. via CTA oder real-time TA). Offene Kommunikationsprozesse sind empfehlenswert, in die Wissenschaft, Unternehmen und zivilgesellschaftliche Organisationen einbezogen sind. Nanotechnologische road-maps können ein geeignetes Integrations- und Orientierungsinstrument sein. Begleitende Ökobilanzierungen als Orientierungshilfe sollten weiter realisiert werden und in den Prozess zurück gespiegelt werden.

- Unternehmen haben eine wesentliche Gestaltungskraft, die verantwortungsvoll genutzt werden muss. Wertschöpfungsübergreifende integrierte Management- und Kommunikationskonzepte sind nanotechnologiebezogen $\mathrm{zu}$ entwickeln. Leitfäden für nachhaltiges Nanodesign gerade auch für kleine und mittlere Unternehmen sind zu entwickeln.

\section{Literatur}

etc-group, 2002: Communiqué: No Small Matter! Nanotech Particles Penetrate Living Cells and Accumulate in Animal Organs. Issue 76. May/June 2002; http://www.etcgroup.org/article.asp?newsid=356, Zugriffsdatum: 19.03.2004

Gleich, A. v., 2004: Leitbildorientierte Technikgestaltung - Nanotechnologie zwischen Vision und Wirklichkeit. In: Böschen, S.; Schneider, M.; Lerf, A. (Hrsg.): Handeln trotz Nichtwissen. Vom Umgang mit Chaos und Risiko in Politik, Industrie und Wissenschaft. Frankfurt a.M., New York: CampusVerlag (erscheint demnächst)

Guston, D.H.; Sarewitz, D., 2001: Real-Time Technology Assessment. In: Technology in Society. 23 (4), S. 98-118

Haum, R.; Petschow, U.; Steinfeldt, M.; Gleich, A. v., 2004: Nanotechnology and Regulation within the framework of the Precautionary Principle. Schriftenreihe des IÖW 173/04. Berlin: Eigenverlag

Joy, B., 2000: Why the future doesn't need us: In: Wired Magazine; http://www.wired.com/wired/ archive/8.04/joy_pr.html, Zugriffsdatum: 19.03.2004

Steinfeldt, M., 2003: Nanotechnologie: Bewertungskriterien und Gestaltungsansatz. Die Nachhaltigkeit der kleinsten Dinge. In: Ökologisches Wirtschaften Heft Nr. 6, S. 20-21

Rip, A.; Misa, T.J.; Schot, J.W. (Hrsg.), 1995: Managing Technology in Society. The Approach of
Constructive Technology Assessment. London: Continuum Intl Pub Group

\section{Kontakt}

Dipl.-Ing. Michael Steinfeldt

Institut für ökologische Wirtschaftsforschung

(IÖW) $\mathrm{gGmbH}$

(Institute for Ecological Economy Research)

Potsdamer Str. 105, 10785 Berlin

Tel.: +49 (0) 30 / 884594 - 18

Fax: +49 (0) 30 / 8825439

E-Mail: michael.steinfeldt@ioew.de

Internet: http://www.ioew.de/ 